\title{
REVIEW
}

\section{Perspectives on Subcutaneous Infliximab for Rheumatic Diseases and Inflammatory Bowel Disease: Before, During, and After the COVID-19 Era}

\author{
Stefan Schreiber · Shomron Ben-Horin • Rieke Alten • René Westhovens • \\ Laurent Peyrin-Biroulet · Silvio Danese · Toshifumi Hibi • \\ Ken Takeuchi · Fernando Magro · Yoorim An · Dong-Hyeon Kim • \\ SangWook Yoon · Walter Reinisch \\ Received: September 27, 2021 / Accepted: November 5, 2021 / Published online: January 6, 2022 \\ (C) The Author(s) 2022, corrected publication [2022]
}

\section{ABSTRACT}

The coronavirus disease 2019 (COVID-19) pandemic has prompted significant changes in

Digital Features This article is published with digital features, including a graphical abstract, to facilitate understanding of the article. To view digital features for this article go to https://doi.org/10.6084/m9.figshare. 17260367.

S. Schreiber

Department of Medicine I, Christian-Albrechts-

University, University Hospital Schleswig-Holstein,

Kiel, Germany

S. Ben-Horin

Gastroenterology Department, Sheba Medical

Center, Tel Aviv University, Tel-Hashomer,

Israel

R. Alten

Department of Internal Medicine II, Rheumatology,

Clinical Immunology, Osteology, Schlosspark

Klinik, University Medicine Berlin, Berlin,

Germany

R. Westhovens

Department of Development and Regeneration,

Skeletal Biology and Engineering Research Center,

Leuven, Belgium

L. Peyrin-Biroulet

Department of Gastroenterology, Nancy University

Hospital, Vandoeuvre-les-Nancy, France

L. Peyrin-Biroulet

Inserm U1256 NGERE, Lorraine University,

Vandoeuvre-les-Nancy, France patient care in rheumatology and gastroenterology, with clinical guidance issued to manage ongoing therapy while minimising the risk

\section{S. Danese}

Gastroenterology and Endoscopy Unit, IRCCS Ospedale San Raffaele, University Vita-Salute San Raffaele, Milan, Italy

T. Hibi

Center for Advanced IBD Research and Treatment, Kitasato Institute Hospital, Kitasato University, Tokyo, Japan

K. Takeuchi

Department of Gastroenterology, IBD Center, Tsujinaka Hospital Kashiwanoha, Chiba, Japan

\section{F. Magro}

Department of Biomedicine, Unit of Pharmacology and Therapeutics, Faculty of Medicine, University of Porto, Porto, Portugal

F. Magro

Department of Gastroenterology, Centro Hospitalar São João, Porto, Portugal

\section{F. Magro}

MedInUP, Centre for Drug Discovery and Innovative Medicines, Porto, Portugal

Y. An · D.-H. Kim · S. Yoon

Celltrion Healthcare Co., Ltd, Incheon, Republic of Korea

W. Reinisch $(\square)$

Division of Gastroenterology and Hepatology,

Department of Internal Medicine III, Medical

University of Vienna, Währinger Gürtel 18-20, 1090

Vienna, Austria

e-mail: walter.reinisch@meduniwien.ac.at 
of nosocomial infection for patients and healthcare professionals (HCPs). Subcutaneous (SC) formulations of biologics enable patients to self-administer treatments at home; however, switching between agents may be undesirable. CT-P13 SC is the first SC formulation of infliximab that received regulatory approval and may be termed a biobetter as it offers significant clinical advantages over intravenous (IV) infliximab, including improved pharmacokinetics and a convenient mode of delivery. Potential benefits in terms of reduced immunogenicity have also been suggested. With a new SC formulation, infliximab provides an additional option for dual formulation, which enables patients to transition from IV to SC administration route without changing agent. Before COVID-19, clinical trials supported the efficacy and safety of switching from IV to SC infliximab for patients with rheumatoid arthritis and inflammatory bowel disease (IBD), and SC infliximab may have been selected on the basis of patient and HCP preferences for SC agents. During the pandemic, patients with rheumatic diseases and IBD have successfully switched from IV to SC infliximab, with some clinical benefits and high levels of patient satisfaction. As patients switched to SC therapeutics, the reduction in resource requirements for IV infusion services may have been particularly welcome given the pandemic, facilitating reorganisation and redeployment in overstretched healthcare systems, alongside pharmacoeconomic benefits and a reduction in exposure to nosocomial infection. Telemedicine and contactless healthcare have been pushed to the forefront during the pandemic, and a lasting shift towards remote patient management and community/home-based drug administration is anticipated. SC infliximab supports the implementation of this paradigm for future improvements of healthcare value delivered. The accumulation of real-world data during the pandemic supports the high level of confidence, with patients, physicians, and healthcare systems benefitting from its uptake. 


\section{Graphical Abstract:}

\section{$\triangle$ Adis}

$\square$ OPEN
$\Omega$ ACCESS

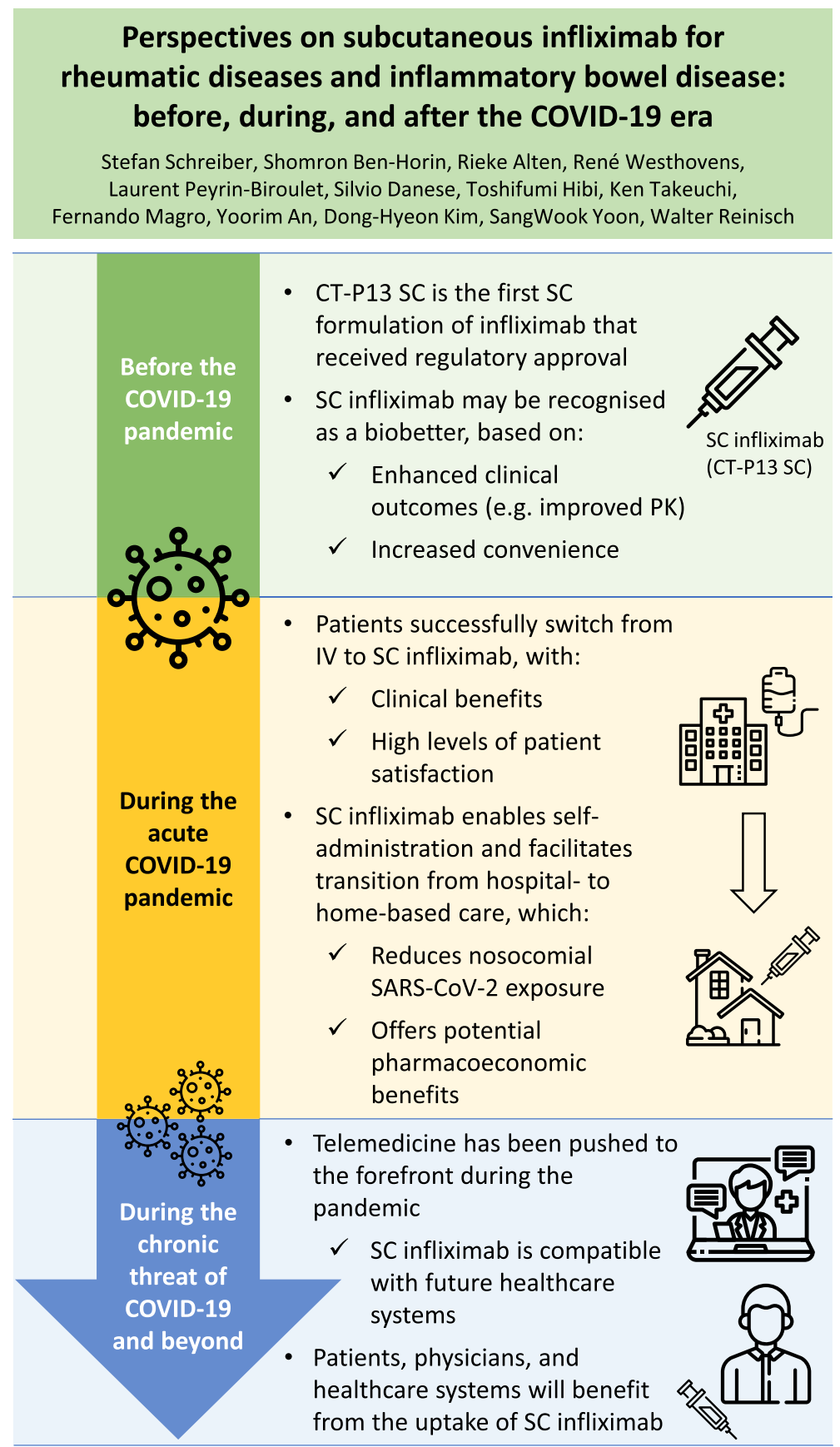

COVID-19, coronavirus disease 2019; IV, intravenous; PK, pharmacokinetics; SARS-CoV-2, severe acute respiratory syndrome coronavirus 2; SC, subcutaneous.

The graphical abstract represents the opinions of the authors. For a full list of declarations, including funding and author disclosure statements, please see the full text online.

(C) The authors, CC-BY-NC 2021. https://doi.org/10.1007/s12325-021-01990-6 
Keywords: Biobetters; Coronavirus disease 2019 (COVID-19); CT-P13 SC; Inflammatory bowel disease; Innovative biologics; Real-world evidence; Rheumatic diseases; Subcutaneous infliximab; Telemedicine

\section{Key Summary Points}

The coronavirus disease 2019 (COVID-19) pandemic has caused a significant burden on healthcare services, including chronic inflammatory diseases such as rheumatic diseases and inflammatory bowel disease (IBD)

The first subcutaneous (SC) formulation of infliximab, CT-P13 SC, offers several advantages over its intravenous (IV) formulation, in terms of an improved pharmacokinetic profile and convenient administration method

Recognition of these 'biobetter' qualities of SC infliximab was growing among physicians and patients prior to the COVID-19 pandemic, tipping their preferences in favour of the SC formulation

Real-world data from patients successfully switching from IV to SC infliximab in rheumatic diseases and IBD settings during the pandemic have supported the clinical findings from the pivotal studies and demonstrated the benefits of SC infliximab for patients, as well as healthcare systems from resource allocation and pharmacoeconomic perspectives

The pandemic has pushed telemedicine and contactless healthcare to the forefront; self-administered biologics like SC infliximab are an important element of remote management of patients with rheumatic diseases and IBD, with SC infliximab expected to remain a cornerstone therapy in the telemedicine concepts beyond the pandemic era

\section{INTRODUCTION}

The coronavirus disease 2019 (COVID-19) pandemic has caused a significant burden on healthcare systems around the world, leading to substantial changes to treatment settings for patients with rheumatic diseases and inflammatory bowel disease (IBD) [1-4]. The highly transmissible nature of severe acute respiratory syndrome coronavirus 2 (SARS-CoV-2) increases the risk of nosocomial infection $[5,6]$; thus, outpatient clinics and hospital attendance have been reduced in order to improve physical distance for those patients who still need to attend in person [3, 4, 7-9]. Minimised patient management via telemedicine has become an important tool in the management of chronically ill patients [3, 4, 7-9]. However, ongoing concerns about acquiring COVID-19 have prompted some patients to avoid appointments or stop the use of parenteral medication [10], although continued treatments are needed to avoid disease flares [4, 7-9].

In the current circumstances, clinicians might particularly welcome novel therapeutic options that are based on highly potent and well-studied molecules, thereby expanding treatment choices for patients with rheumatic diseases and IBD $[11,12]$. Recent technological advances have driven the development of innovation in biologics through other modifications, such as the first subcutaneous (SC) formulation of infliximab, CT-P13 SC. Technological innovation has resulted in a biobetter status for CT-P13 SC (and hence designation as a value-added medicine or a biobetter), as it offers significant clinical benefits including improved pharmacokinetics (PK) and a more convenient mode of delivery compared with intravenous (IV) infliximab [13, 14]. Availability of CT-P13 SC may have facilitated treatment changes during the pandemic, allowing a shift in administration route from IV to SC, and correspondingly, from in-clinic to at-home treatment, mitigating the risk of nosocomial or commute-related SARS-CoV-2 exposure $[15,16]$. Indeed, patients with rheumatic diseases and IBD have successfully switched from IV to SC infliximab during the pivotal studies and the 
COVID-19 pandemic [15-22]. With the renewed interest in SC administration of biologics, we discuss the clinical implications and advantages of SC infliximab in the treatment of rheumatic diseases and IBD during the pandemic and beyond. This article is based on previously conducted studies and does not contain any new studies with human participants or animals performed by any of the authors.

\section{THE ADVENT OF SC INFLIXIMAB}

Infliximab is an example of how innovation has led to treatment evolution and improvementsfrom the introduction of the reference product in 1998, followed by the approval of the first infliximab biosimilar (CT-P13) in 2013, and the approval of CT-P13 SC in Europe in 2019 [13]. Infliximab is the most widely studied and used anti-tumour necrosis factor (TNF) biologic for therapy of chronic immune-mediated diseases [23-25]. Before the introduction of these biosimilar products, 50 manufacturing process changes were recorded for reference infliximab between European Union registration in 1999 and October 2014 [26]. Manufacturing changes of a product may lead to a possible reduction in side effects and immunogenicity $[27,28]$. Since such modifications can impact the clinical attributes of a molecule, comparability exercises are required to ensure quality, efficacy, and safety after a manufacturing process change, with experience of these assessments forming the foundation for regulatory agencies to evaluate biosimilarity [29].

As well as being the first infliximab biosimilar to receive regulatory approval from the European Medicines Agency (EMA) and United States (US) Food and Drug Administration (FDA) [13], CT-P13 was recognised as the first biosimilar monoclonal antibody in Japan and South Korea [30, 31]. Global regulatory acceptance was based on the pivotal PLANETRA and PLANETAS studies in patients with rheumatoid arthritis (RA) and ankylosing spondylitis (AS), respectively $[32,33]$. Although not required for the approval process, further indications were supported by the randomised PLANETCD study in the gastroenterology setting [34]. Following pivotal studies conducted in patients with RA and IBD $[18,19]$, CT-P13 SC received regulatory approval from the EMA for RA in 2019 [35], and for the other infliximab indications in adults, comprising AS, psoriatic arthritis (PsA), psoriasis, Crohn's disease (CD), and ulcerative colitis (UC), in 2020 [36, 37]. In North America, CT-P13 SC received approval for the RA indication in Canada in 2021 [38]. The FDA is considering CT-P13 SC under the new drug pathway because of the difference in dose and administration route, as well as PK benefits, compared to its IV formulation [14, 39]. Therefore, the FDA has requested that randomised, placebo-controlled efficacy studies are conducted. Studies are underway in patients with moderately to severely active CD (ClinicalTrials.gov Identifier NCT03945019) and UC (NCT04205643), with FDA approval anticipated in the second quarter of 2022 [39]. Given the new drug status, it is unclear whether the FDA will allow extrapolation of use to all indications or whether studies will be needed for each disease area [39].

For CT-P13 SC, two pivotal studies demonstrated non-inferiority to CT-P13 IV in terms of efficacy (in part 2 of the phase I/III CT-P13 SC 3.5 study in patients with RA) [18] and PK (in part 2 of the phase I CT-P13 SC 1.6 study in patients with IBD) [19]. CT-P13 SC provided a more stable drug exposure than CT-P13 IV in both trials, with levels consistently higher than the target therapeutic concentration, representing a pharmacological advantage for CT-P13 SC [11, 13, 18, 19]. While the pivotal studies included CT-P13 IV dose loading prior to week 6 , predicted exposure and efficacy were comparable between the CT-P13 SC and CT-P13 IV arms from week 6 onwards. The Committee for Medicinal Products for Human Use recommends that CT-P13 SC can be initiated without IV dose loading in patients with RA [40], which could potentially further reduce time associated with drug administration, reduce healthcare professional (HCP) time, and improve flexibility for patients.

Since it is a chimeric antibody, infliximab can induce the formation of anti-drug antibodies (ADAs), contributing to a loss of response [41]. While a general perception exists that SC 
biotherapeutics are potentially more immunogenic than those administered via the IV route $[13,42]$, the pivotal CT-P13 SC studies refuted this, as similar, albeit numerically lower, immunogenicity was observed for SC- versus IVadministered CT-P13 $[18,19]$. A post hoc analysis of pivotal data in patients with RA and CD also demonstrated significantly lower immunogenicity for patients receiving CT-P13 SC than those receiving CT-P13 IV $(p<0.0001)$ [43]. The proportion of patients who converted to neutralising antibody-positive status was also lower for SC-versus IV-administered CT-P13 in the IBD study [19]. From an immune mechanistic perspective, the higher trough concentrations observed with CT-P13 SC may putatively induce high-zone tolerance, resulting in immune downregulation towards the agent and reduced immunogenicity [13]. An alternative or complementary mechanism may be decreased formation of drug-antigen immune complexes in the context of high drug levels, possibly leading to lower immune activation towards the drug [44].

The enhanced pharmacological and clinical outcomes observed with CT-P13 SC compared with IV infliximab have, in part, promoted the development of a new definition for biobetters. An international Delphi consensus meeting involving gastroenterologists and rheumatologists agreed that a "biobetter is a modified version of a specific approved biologic that enhances clinical outcomes (e.g., improved efficacy) and/or drug pharmacology (e.g., PK and/or pharmacodynamics)", citing CT-P13 SC in support of the new definition [14]. Further to the PK benefit discussed at the consensus meeting [14], recent meta-analyses have suggested that CT-P13 SC offers an improved benefit-to-harm ratio in patients with RA, compared with IV infliximab $[45,46]$.

\section{CLINICAL EVIDENCE AND IMPLICATIONS OF SC INFLIXIMAB PRIOR TO THE COVID-19 PANDEMIC}

SC self-application of peptide/protein-based medications is not new: pens for injection of insulin in the therapy of diabetes mellitus have been around for over 30 years [47]. In rheumatic diseases and in the IBD setting, several therapeutic agents can be primarily administered by SC injection [48-50]. However, the development of SC infliximab has shifted the landscape for anti-TNF therapy, among further advantages (Table 1). In the past, physicians could use infliximab for continuous IV therapy and adalimumab for continuous SC therapy in IBD [51], with additional SC anti-TNF options (including etanercept and golimumab) available for rheumatic diseases [42]. With only a few anti-TNF biologics available with dual formulations, a combination of IV loading and SC maintenance dosing was not a readily available option, although it may carry significant advantages for patients (including high peak serum drug levels with IV induction and constant serum drug levels with SC maintenance) $[35,52]$.

Prior to the COVID-19 pandemic, SC antiTNF agents were employed depending on patient preferences and physician recommendations [51], with surveys suggesting that both patients and physicians in rheumatology and gastroenterology settings prefer SC to IV biologics [53-55]. A survey of patients and HCPs from rheumatology clinics in Denmark revealed that $71 \%$ of patients currently self-injecting their treatment at home, $77 \%$ of biologic-naïve patients, and $87 \%$ of HCPs preferred the SC route of administration [53]. The majority of patients in the survey who were currently receiving SC anti-TNF agents had previously received an IV biologic, providing a useful insight into the perspectives of patients who had switched administration route [53]. In another study of 25 TNF inhibitor-naïve patients, $60 \%$ chose to receive an SC biologic (adalimumab) over an IV biologic (infliximab) for reasons related to the route of administration rather than the drug itself [54]. Similarly, when TNF inhibitor-naïve patients with CD were asked to choose an anti-TNF therapy, approximately two-thirds opted for a treatment that was delivered by SC injection [55]. Patients have cited ease of use, convenience, and time taken for administration as important factors in treatment selection [53-55]. In addition, SC medications may offer more flexibility for 
Table 1 Clinical implications of SC infliximab before, during, and beyond the COVID-19 pandemic era

\section{Clinical implications of SC infliximab}

\section{Before the COVID-19 pandemic}

SC infliximab offers comparable efficacy and safety to IV infliximab, with an improved PK profile: these clinical advantages mean SC infliximab has been recognised as an example of a biobetter

SC infliximab may also offer lower immunogenicity than IV infliximab in patients with either RA or IBD

The increased convenience of SC infliximab could provide a convenient and empowering alternative to IV infliximab, which may be preferred by patients

The flexibility and convenience of SC infliximab may afford patients greater control over their treatment, potentially improving adherence

\section{During the acute COVID-19 pandemic}

Successful outcomes after switching from IV to SC infliximab in pivotal studies have been reproduced in real-world rheumatology and gastroenterology settings during the pandemic, with benefits in terms of PK, convenience, and patient preference observed

SC infliximab facilitates a shift from hospital to home-based care as patients can transition from IV to SC administration routes without changing therapeutic agent

Reduced hospital attendance due to uptake of SC biologics lowers the risk of nosocomial exposure to SARS-CoV-2 for HCPs and patients

Healthcare resource requirements are reduced with patients switching from IV to SC infliximab, facilitating reorganisation and redeployment during the surge in demand brought about by the pandemic, as well as offering cost savings

Ongoing home-based self-administration of SC infliximab may be associated with substantial long-term cost savings for healthcare systems

\section{During the chronic threat of COVID-19 and beyond}

SC infliximab is compatible with remote patient management via telemedicine and contactless healthcare approaches, which are expected to become embedded in healthcare systems post-pandemic

Accumulating real-world data should improve confidence and uptake for SC infliximab

Patient education, combined with suitable remote monitoring of disease activity, drug levels, and compliance, will be required to ensure safe and adherent long-term treatment with SC infliximab

COVID-19 coronavirus disease 2019, HCP healthcare professional, IBD inflammatory bowel disease, $I V$ intravenous, $S A R S-C o V-2$ severe acute respiratory syndrome coronavirus $2, P K$ pharmacokinetic(s), $R A$ rheumatoid arthritis, $S C$ subcutaneous

patients wishing to travel-the availability of medications abroad, particularly IV infusions, can pose a major obstacle and requires advance planning [56-58]. The availability of SC infliximab now enables a change in administration route from IV to SC with the same agent, depending on the requirement for drug exposure, safety of the administration environment, and convenience. 


\section{CLINICAL IMPLICATIONS OF SC INFLIXIMAB IN THE COVID-19 PANDEMIC ERA AND BEYOND}

\section{During the Acute COVID-19 Pandemic}

During the initial phase of the COVID-19 pandemic, SC infliximab offered several benefits for patients and healthcare systems, including reducing hospital attendance and the healthcare resource burden. This provided potentially positive impacts from a pharmacoeconomic perspective, alongside clinical benefits for patients when switching. In addition, SC infliximab may have other positive effects on COVID-19 infection through its mechanism of action as an anti-TNF agent.

\section{SC Infliximab Provides Opportunities to Reduce Hospital Attendance Without Changing Biologic}

Several clinical societies and institutions developed clinical recommendations and guidelines in response to the pandemic, providing guidance on patient management while reducing potential nosocomial exposure to SARS-CoV-2 for both HCPs and patients through minimising hospital visits (Table 2) [4, 7-9, 59-64]. While patients were advised to continue taking their medications [4, 7-9, 59-64], some guidelines recommended prioritising the use of SC biologics above IV formulations $[9,60,62]$ or to consider the route of administration when making treatment decisions [61]. Other guidelines advised against treatment changes from IV to SC biologics solely because of the pandemic situation $[4,8,65]$. However, it was recognised that a switch to SC biologics might be needed if it was not possible to continue infusion services safely [8]. The availability of IV and SC formulations of CT-P13 allows patients who receive IV infliximab to switch to the SC route without changing biologic, in compliance with these guidelines. This avoids a potentially undesired switch of therapeutic agent from IV infliximab to SC adalimumab, which has been associated with an increased risk of a flare in patients with CD [66], as noted in European Crohn's and Colitis Organization guidelines $[8,65]$. In addition to the uptake of SC formulations, efforts to avoid nosocomial infection included the use of telemedicine for consultations and reducing the frequency of blood monitoring [4, 7-9, 59-61, 63, 64]. For patients with IBD, non-emergency endoscopies were postponed $[4,8,63,64]$. As real-world data accumulated during the pandemic, it was clear that disease management was evolving rapidly, highlighting the importance of shared clinical decisionmaking between physician and patient.

\section{Switching from IV to SC Infliximab During the COVID-19 Pandemic}

The pivotal studies evaluating CT-P13 SC demonstrated that efficacy was comparable for patients with UC, CD, or RA who received CT-P13 SC throughout (after dose loading) or who switched from CT-P13 IV to CT-P13 SC from week 30 [18, 19]. Other than an anticipated increase in local site pain following the switch to CT-P13 SC (the extent of which reduced with repeated injections), safety was similar between treatment arms in both studies. These results suggested that switching from CTP13 IV maintenance treatment to SC infliximab was feasible during established remission. In addition, trough drug concentrations increased in the CT-P13 IV groups following the switch to CT-P13 SC, becoming similar to concentrations maintained in patients receiving CT-P13 SC throughout.

During the COVID-19 pandemic, switching patients to SC therapies offered several potential benefits and successful approaches have been shared $[22,67,68]$. For SC infliximab, real-world experience (or expert opinion) has been accumulating to support switching from IV to SC formulations. Reports of pandemic-driven initiatives have demonstrated that switching from IV infliximab to CT-P13 SC has been well tolerated and clinically favourable in patients with IBD [16, 17, 22, 69]. In response to the pandemic, a programme in which stable patients with IBD were switched from IV infliximab to CT-P13 SC was initiated in two UK hospitals $[16,70]$. The most recent report noted that 172 patients had switched to CT-P13 SC since April 2020, with high levels of patient satisfaction [70]. Of 88 randomly selected patients surveyed, 
Table 2 Selected key messages of clinical guidelines in rheumatic diseases and inflammatory bowel disease in the COVID-19 pandemic era, with an emphasis on treatment changes and switching to SC therapies

Guideline Key messages for patients not known or suspected to be infected with SARS-CoV-2

References

\section{Rheumatic diseases}

ACR Ongoing treatment (hydroxychloroquine/chloroquine, sulfasalazine, methotrexate, leflunomide, immunosuppressants [e.g. tacrolimus, cyclosporin A, mycophenolate mofetil, azathioprine], biologics, JAK inhibitors and NSAIDs) in patients with stable rheumatic disease may be continued. Denosumab may still be given, extending dose intervals if necessary to no longer than every 8 months

Measures such as reduced frequency of laboratory monitoring, use of telemedicine, and increased dosing intervals for IV therapies may be reasonable to reduce healthcare encounters and potential exposure to SARS-CoV-2

AFLAR Medications for rheumatic diseases should be continued as normal as there is no evidence of increased SARS-CoV-2 infection risk in patients with RMDs or receiving DMARDs

Limit hospital attendance by considering use of SC formulations of bDMARDs and bsDMARDs instead of IV infusions

The following should be considered by physicians to reduce patients' hospital attendance: less frequent blood monitoring (for stable patients), longer prescription periods, and virtual clinics

EULAR Treatments should be continued unchanged (such as NSAIDs, glucocorticoids, sDMARDs, bDMARDs, osteoporosis medications, and analgesics)

Regular blood monitoring and face-to-face rheumatology consultations can be postponed for stable patients, and remote consultations can be used where necessary

NICE NSAIDs and denosumab do not need to be stopped. Treatment with zoledronate can be postponed for up to 6 months. Prednisolone should not be stopped suddenly. Use oral corticosteroids where possible

Consider switching patients receiving IV biologics to an SC formulation of the same treatment; if this is not possible, discuss changing to an alternative SC treatment with the patient

Assess whether the frequency of IV immunoglobulins can be reduced

Minimise face-to-face contact by avoiding non-essential face-to-face consultations, offering telephone or video consultations, using medication delivery services, and expanding communitybased blood monitoring

Consider increasing intervals between blood tests for drug monitoring (where safe)

SFR Maintain effective and well-tolerated treatments (such as methotrexate, leflunomide, sulfasalazine, bDMARDs) to avoid potential disease flares

There are no contraindications to initiating or maintaining NSAIDs or JAK inhibitors

Minimise dose of oral corticosteroids to $\leq 10 \mathrm{mg}$ per day if possible

Consider switching patients receiving IV biologics to their SC formulations to avoid hospital attendance, for patients who can self-administer treatment 
Table 2 continued

Guideline Key messages for patients not known or suspected to be infected with SARS-CoV-2

\section{Inflammatory bowel disease}

AGA To avoid relapse (due to non-adherence), patients should maintain their current regimens

Elective switching of IV medications (e.g., infliximab) to SC therapies (e.g., adalimumab) or home infusions for IV medications is not recommended

Only urgent and emergent endoscopic procedures should take place

BSG Patients should continue their current medications. Access to injectable treatment (infliximab, vedolizumab, ustekinumab, adalimumab, certolizumab and golimumab) should be maintained. Corticosteroids should be avoided if possible but not stopped suddenly

Access to and home care provision of SC medicines should be prioritised, with infusion suite services maintained to prevent disease flares and hospital admission; enforced switching from IV to SC therapies is not recommended

Monitor disease activity remotely using virtual clinics, with blood tests conducted at non-hospital sites; routine blood monitoring may be deferred

Non-emergency endoscopy should not take place; routine elective operations and complex surgeries should be deferred where possible

ECCO Continue treatment with immunomodulators, biologics, and JAK inhibitors

Do not switch stable patients from IV infliximab to SC adalimumab unless it is not possible to provide IV infusions; switching to SC therapies may be considered where it is not possible to run an infusion service safely

Implement telemedicine and remote monitoring; only conduct appointments for decision-making

Postpone non-urgent endoscopic procedures and limit hospitalisation and surgery to lifethreatening situations

IOIBD Patients should not reduce the dose or discontinue anti-TNF therapies, thiopurines, 5-aminosalicylic acid, budesonide, methotrexate, vedolizumab, ustekinumab, or tofacitinib. Patients taking prednisone therapy $(\geq 20 \mathrm{mg} /$ day) should reduce the dose of therapy to prevent SARS-CoV-2 infection

Postpone elective surgery and non-essential endoscopic procedures 
Table 2 continued

\begin{tabular}{lll}
\hline Guideline & Key messages for patients not known or suspected to be infected with SARS-CoV-2 & References \\
\hline NICE & $\begin{array}{l}\text { Continue existing courses of treatment to minimise the risk of disease flares but consider whether } \\
\text { any changes are needed to minimise face-to-face contact during the COVID-19 pandemic, } \\
\text { including to the route of administration and mode of delivery }\end{array}$ \\
& When deciding whether to start a new treatment, consider factors including whether there is a \\
route of administration that could make hospital attendance or admission less likely \\
Minimise contact by avoiding face-to-face consultations that are not essential, offering \\
consultations via telephone or video, using medication delivery services, and expanding \\
community-based blood monitoring
\end{tabular}

$A C R$ American College of Rheumatology, AFLAR African League Against Rheumatism, AGA American Gastroenterological Association, (b/bs)DMARD (biologic/biosimilar) disease-modifying anti-rheumatic drug, BSG British Society of Gastroenterology, COVID-19 coronavirus disease 2019, ECCO European Crohn's and Colitis Organization, EULAR European Alliance of Associations for Rheumatology, IOIBD International Organization for the Study of Inflammatory Bowel Diseases, $I V$ intravenous, JAK Janus kinase, NICE National Institute for Health and Care Excellence, NSAID nonsteroidal anti-inflammatory drug, $R M D$ rheumatic and musculoskeletal disease, $S A R S-C o V-2$ severe acute respiratory syndrome coronavirus 2, SC subcutaneous, $s D M A R D$ synthetic disease-modifying anti-rheumatic drug, SFR French Society of Rheumatology, $T N F$ tumour necrosis factor

$85 \%$ agreed they were happier on SC infliximab than IV infliximab, and $92 \%$ and $86 \%$ reported that SC infliximab was easy and felt safe to use, respectively [70]. A previous report after 163 patients had switched found that trough drug concentrations were similar or higher after the switch than before [16]. Mean infliximab levels were significantly higher 3 months post-switch than at baseline, and were maintained 6 months post-switch [70]. SC infliximab was well tolerated with low rates of adverse events $[16,70]$. A further observational study evaluated switching from CT-P13 IV to CT-P13 SC in 17 patients with IBD who were in clinical remission [22]. Serum drug concentrations were significantly higher 6 months post-switch than at baseline, with decreased clinical Mayo scores and faecal calprotectin levels also observed. Taken together, these findings suggest that results from the pivotal studies, such as the improved PK profile following the switch to CT-P13 SC, are reproducible in real-world settings, demonstrating the reliability of the pivotal data for CT-P13 SC.

In addition, on the basis of our own clinical experiences, rheumatologists are also reporting successful switching to CT-P13 SC in individual patients with AS, RA, and PsA; CT-P13 SC is well tolerated, with no indications of an increase in disease activity, and patients appreciate the reduction in hospital visits during the pandemic [20]. For patients, homecare-based management can increase independence, which can have a positive impact on quality of life, making everyday activities easier and providing a sense of freedom [71-73]. By allowing patients to take charge of their own therapy with self-administration, we believe SC therapeutics may also give patients a sense of empowerment that provides comfort in this era of insecurity.

\section{Self-Administered Therapies Reduce the Healthcare Resource Burden During the Pandemic}

Minimising hospital visits per clinical guidelines reduced both the infection risk for patients [16] and the burden on overstretched medical facilities. The reduction in healthcare resources concomitant with reduced administration of IV therapies could facilitate reorganisation and redeployment as required during the unprecedented demand on medical services [2, 11, 42]. Experts have agreed that SC biobetters could play an important role during health 
emergencies, such as the COVID-19 pandemic, although decisions to switch must be shared between patient and physician [14].

\section{SC Infliximab Offers Potential Pharmacoeconomic Benefits}

Switching from IV to SC infliximab can also have a beneficial pharmacoeconomic impactan important consideration for healthcare facilities given the pandemic-related surge in demand. A UK analysis in RA and IBD found that the annual costs for SC infliximab treatment could be approximately 50\% lower than those for IV infliximab ( $£ 1457$ versus $£ 2867$ ) [74]. The study also identified savings for patients through reduced hospital parking charges and lost personal time, and increased productivity [74]. Given that the shift to community/home-based drug administration is likely to be maintained in the future, SC biologics may be more economically sustainable than IV treatments: with up to $15 \%$ of the cost of an IV therapeutic coming from the infusion itself, long-term savings could be substantial [39]. A budget impact analysis from the UK payer perspective estimated that, over 5 years, the introduction of CT-P13 SC could result in nearly $£ 40$ million of cost savings, which could enable an additional 4466 patients to receive SC infliximab [75]. Since patients can lose response to IV infliximab over time and, consequently, may need to receive higher or more frequent doses, the cost savings with SC administration could be even higher [39].

\section{Other Potential Clinical Benefits of Anti-TNF Treatment for Patients with COVID-19}

Initially, the impact of COVID-19 on patients with chronic autoinflammatory diseases was unknown [76], including the SARS-CoV-2 infection risk of patients receiving immunomodulatory/immunosuppressive treatments [77, 78]. Meta-analyses have indicated a higher prevalence of COVID-19 in patients with autoimmune diseases compared with control patients or the general population, without an increased risk of hospitalisation, intensive care unit admission, or death [77, 79]. When analysed by disease, the prevalence of COVID-19 was higher in RA (0.009) than IBD (0.003), with higher prevalence associated with glucocorticoid use [77].

The immunosuppressive effects of anti-TNF therapy were initially hypothesised to put patients at high risk of SARS-CoV-2 infection and for development of severe forms of the disease; however, this was by and large refuted by ensuing evidence that rates of severe infection were not increased, with an inverse relationship suggested between anti-TNF treatment and hospitalisation or mortality due to COVID-19 (Table 3; 76-84]). Evidence from the SECURE-IBD registry showed a lower risk of severe COVID-19 with anti-TNF agents versus corticosteroids (adjusted odds ratio 0.9 versus 6.9) [78]. Moreover, the COVID-19 Global Rheumatology Alliance physician-reported registry reported an inverse association between anti-TNF treatment and hospitalisation with COVID-19 and a higher risk of hospitalisation with moderate to high glucocorticoid use versus no glucocorticoid use [82]. These results were supported by a meta-analysis in patients with rheumatic diseases, which reported a lower hospitalisation risk for patients receiving antiTNF agents (odds ratio 0.38) [79]. Registry findings have also linked corticosteroid/thiopurine use with severe COVID-19, and identified an increased risk of severe COVID-19 in patients receiving both thiopurines and anti-TNF agents versus anti-TNF monotherapy [76, 78]. This may make SC infliximab especially appealing during the pandemic given its efficacy in the pivotal IBD study, in which two-thirds of patients were not receiving concomitant corticosteroids [19]. Furthermore, a recent analysis demonstrated comparable clinical efficacy and PK for patients who received SC infliximab with or without concomitant oral immunomodulators (azathioprine, 6-mercaptopurine, or methotrexate) [85]. Since combination therapy appears to exert its effect through improving the PK profile of infliximab, concomitant immunomodulators may not be required to achieve clinical targets if higher exposure can be delivered with the biologic itself [86]. Such potential benefits may be realised with SC infliximab owing to its improved PK profile. 
Table 3 Clinical impact of anti-TNF treatment on SARS-CoV-2 infection

\begin{tabular}{lc}
\hline Clinical impact of anti-TNF treatment & References \\
\hline $\begin{array}{l}\text { No increase in rates of severe SARS-CoV-2 infection identified in patients with RA or IBD treated with } \\
\text { anti-TNF agents }\end{array}$ & 79-81] \\
Inverse relationship between anti-TNF therapy and hospitalisation or mortality due to COVID-19 suggested & {$[76-79,82]$} \\
by meta-analyses and registry studies for patients with rheumatic diseases or IBD & [83] \\
The anti-inflammatory effects of infliximab may help to combat the severe COVID-19-induced cytokine \\
storm and therefore help to treat COVID-19 \\
Seroconversion occurs in most infliximab-treated patients after two SARS-CoV-2 vaccine doses
\end{tabular}

COVID-19 coronavirus disease 2019, IBD inflammatory bowel disease, $R A$ rheumatoid arthritis, $S A R S$-CoV-2 severe acute respiratory syndrome coronavirus $2, T N F$ tumour necrosis factor

The potentially protective effect of anti-TNF agents against COVID-19 may be related to their effects on the entry receptor angiotensinconverting enzyme 2 (ACE2) and reductions in circulating TNF levels [80, 81]. Patients with IBD taking TNF inhibitors have significantly lower gut ACE2 expression than do patients on no medication [87]. Anti-TNF agents may also help to neutralise the high serum $\mathrm{TNF} \alpha$ concentrations that have been associated with more severe COVID-19 [81]. Indeed, evidence from a case series of patients with COVID-19 (but without IBD) has suggested that infliximab may help to combat severe COVID-19-induced cytokine storm syndrome by reducing systemic inflammation [83]. In addition, an observational study of Italian patients with IBD has reported that non-gut-selective agents (such as anti-TNF therapies) were associated with a lower incidence of SARS-CoV-2 infection, symptomatic COVID-19, and hospitalisations than were gut-selective biologics [88]. Consequently, there have been calls to investigate anti-TNF therapies as treatments for COVID-19 in clinical trials [89]: infliximab has recently been announced as one of three agents to be tested in hospitalised patients with COVID-19 in the World Health Organization's Solidarity PLUS trial [90].

Clinical trials and cohort studies have also assessed the potential benefits of various other biologic therapies for the treatment of COVID-19 [91, 92]. Immunotherapeutic approaches have aimed to improve patient outcomes through combatting the SARS-CoV2-induced cytokine storm, with tocilizumab (targeting interleukin [IL]-6) and anakinra (targeting IL-1) among the biologics evaluated [91]. Janus kinase (JAK) inhibitors are another important class of agents demonstrating efficacy against COVID-19 [93, 94]; however, a pooled analysis of data from registries for inflammatory arthritis, IBD, and psoriasis found that patients receiving JAK inhibitor monotherapy had higher odds of hospitalisation or death associated with COVID-19 than those receiving monotherapy with anti-TNF agents [95]. Neutralising monoclonal antibodies targeting the SARS-CoV-2 receptor binding domain have also been developed, directly inhibiting viral entry into human cells [92]. Such agents include casirivimab, imdevimab, bamlanivimab, etesevimab, and regdanvimab (CT-P59), which have received full or emergency use authorisations from regulatory authorities for the treatment of patients with COVID-19 [92, 96].

Protective immunity and vaccination will be critical to ending the COVID-19 pandemic, and a global vaccination programme is underway. Some evidence suggests TNF inhibitors may impair immune responses to pneumococcal, influenza, and viral hepatitis vaccinations [97]; thus, it is important to understand their impact in the context of COVID-19. The UK-based CLARITY study has shown that infliximab 
attenuated the serological response after natural infection, finding an inverse relationship between infliximab trough levels and seroconversion $[84,97]$. Lower seroconversion rates in infliximab-treated patients were also noted after a single SARS-CoV- 2 vaccine dose, which could increase the likelihood of recurrent SARS-CoV-2 infection in affected patients [97]. However, it is important to view these findings in the context of the similar or reduced risk of severe COVID-19 with anti-TNF treatment, as discussed previously [76-81]. Furthermore, infliximab- and vedolizumab-treated patients who had received their second SARS-CoV-2 vaccine dose (as recommended by health authorities) or were vaccinated following natural infection had comparable seroconversion rates $(85 \%$ and $86 \%$, respectively) [84]. By comparison, $79 \%$ of anti-TNF-treated patients with psoriasis seroconverted after one dose of a SARS-CoV-2 vaccine, compared with only $47 \%$ of patients receiving methotrexate [98].

\section{Chronic Threat of COVID-19 and Beyond}

While we have discussed SC infliximab in context of the COVID-19 pandemic in this article, it is important to note that many of its benefits will continue to positively impact patients and healthcare systems beyond the pandemic era. This includes the clinical benefits of SC infliximab, such as the improved PK profile, the pharmacoeconomic benefits for healthcare systems, and the improved convenience of treatment for patients. In addition to this, it is important to consider the role SC infliximab could play in healthcare systems as they evolve for the future.

While remote patient management systems were already being evaluated in rheumatic diseases and IBD, the pandemic has accelerated their uptake and pushed telemedicine (often termed telerheumatology in the rheumatic disease setting) to the forefront, allowing both HCPs and patients to become more familiar with the concept $[2,69,99-103]$. Before the pandemic, home-based automated systems and web-based services were developed to encompass telemonitoring, teleconsulting, and tele- education for patients with IBD [104], with patient acceptance of virtual monitoring increasing in rheumatology and gastroenterology settings [104, 105]. One systematic review found telerheumatology to be met with high rates of patient satisfaction [106], although potential barriers, such as accessibility and acceptance of telemedicine by older patients, must be considered [107]. Indeed, rheumatology patients receiving SC biologics have been managed successfully via telemedicine during the pandemic $[2,108]$. In Italy, a retrospective study of patients receiving SC or oral therapies for RA, PsA, or AS reported no significant differences in outcomes between those monitored remotely or attending hospital visits [108]. Similarly, US surveys revealed that medication interruptions were more common in patients who avoided the clinic and did not have access to telemedicine $[10,109]$. In IBD, significantly improved medication adherence and reductions in outpatient visits and hospitalisations were reported with telemedicine versus standard care prior to the pandemic [110, 111]. During the pandemic, a UK survey reported that telephone consultations replaced $86 \%$ of face-to-face clinics for patients with IBD [112]; in one unit, 92\% of patients who had a consultation via telephone rather than in person were satisfied with their experience [102]. Gastroenterologists have also reported patient preferences for virtual management approaches during the pandemic and have described the value of SC biologics, including CT-P13 SC, in delivering home carebased management [71].

In the future, the management of patients receiving SC biologics, including CT-P13 SC, should be accompanied by remote patient monitoring where digital tools (such as mobile applications or wearables tracking patients' physiology and physical behaviour) are used to monitor disease activity status, inflammatory burden, and drug exposure to inform clinical care [113]. In IBD, new applications that can complement the transition from symptombased disease management to inflammationbased care are anticipated [114]. Faecal calprotectin measurements could be used as part of tight control or treat-to-target strategies for anti-TNF dose optimisation in patients with CD 
[115], with digital tracking potentially used to assess disease activity and escalate therapy if needed [114]. Digital therapeutic drug monitoring (TDM) for anti-TNF therapy could also benefit patients; TDM enables dosage adjustments based on serum drug and ADA concentrations to maintain therapeutic target drug levels $[116,117]$. The more stable serum drug levels seen with SC administration could potentially allow for any day sampling, unlike the strict pre-dose sampling required for IV-administered biologics: studies with SC-administered adalimumab support this theory $[118,119]$. This may make blood test scheduling easier and more convenient for patients. Cost savings have also been identified with TDM of infliximab treatment in patients with IBD $[120,121]$.

Telemedicine and contactless healthcare are expected to continue to grow post-pandemic as key concepts of future healthcare systems (Table 1) [2, 69, 99-102]. Indeed, acceptance of digital tools increased during the pandemic [105]. Although long-term administration of medications can lead to a lack of adherence, which can be challenging to monitor for drugs that are self-administered at home [122], patient education can improve compliance with SC anti-TNF therapy [123]. While some physicians may be concerned about patient adherence to SC therapies, high adherence rates with SC anti-TNF agents have been reported in a Canadian analysis of patients with RA, AS, or PsA [124]. Combined with the IV formulation, CT-P13 SC may afford patients more control over their treatment [125], with increased flexibility and convenience [13]. In turn, this could improve adherence: a Spanish observational study in RA concluded that compliance could be increased with less complex regimens [126]. HCPs must ensure that patients receive sufficient information and guidance to safely continue to self-administer SC therapeutics, including CT-P13 SC, over the long-term. CTP13 SC administration, when combined with a good monitoring tool for TDM and appropriate patient education, is suitable for remote usage and is compatible with this future paradigm. This article is based on previously conducted studies and does not contain any new studies with human participants or animals performed by any of the authors.

\section{CONCLUSION}

The advent of SC infliximab marks an innovation in the treatment landscape for rheumatic diseases and IBD, offering patients and physicians numerous benefits that have been particularly welcome during the COVID-19 pandemic (Table 1). As accumulating real-world data improve confidence in the use of SC infliximab, its benefits are expected to be realised far beyond the pandemic as SC infliximab continues to be integrated into the healthcare systems of the future.

\section{ACKNOWLEDGEMENTS}

Funding. Open access funding was provided by Medical University of Vienna. The development and publication of this article was funded by Celltrion Healthcare Co., Ltd (Incheon, Republic of Korea).

Medical Writing and/or Editorial Assistance. Medical writing support was provided by Beatrice Tyrrell, DPhil, at Aspire Scientific Limited (Bollington, UK).

Authorship. All named authors meet the International Committee of Medical Journal Editors (ICMJE) criteria for authorship for this article, take responsibility for the integrity of the work as a whole, and have given their approval for this version to be published.

Author Contributions. All authors (Stefan Schreiber, Shomron Ben-Horin, Rieke Alten, René Westhovens, Laurent Peyrin-Biroulet, Silvio Danese, Toshifumi Hibi, Ken Takeuchi, Fernando Magro, Yoorim An, Dong-Hyeon Kim, SangWook Yoon, and Walter Reinisch) contributed to the concept and development of the article, from the outline to subsequent drafts. All authors have read and approved the final manuscript. 
Disclosures. Stefan Schreiber has received honoraria for consulting and/or lectures from AbbVie, Allergan, Amgen, AMT, Arena, Biogen, Boehringer, Bristol Myers Squibb, Celgene, Celltrion, Falk, Galapagos, Gilead, Janssen, Lilly, Merck, Pfizer, Roche, Takeda, and Tillotts. Shomron Ben-Horin has received consultancy/ advisory board fees from AbbVie, Celltrion, Falk, Ferring, Galmed, GSK, Janssen, Pfizer, Takeda, and Novartis; and research support from AbbVie, Celltrion, Galmed, Janssen, Pfizer, and Takeda. Rieke Alten has received speaker fees from Celltrion. René Westhovens has acted as an advisor, speaker, and principal investigator for Celltrion, Galapagos, and Gilead. Laurent Peyrin-Biroulet has received personal fees from AbbVie, Allergan, Alma, Amgen, Applied Molecular Transport, Arena, Biogen, Boehringer Ingelheim, Bristol Myers Squibb, Enterome, Enthera, Celgene, Celltrion, Ferring, Fresenius Kabi, Galapagos, Genentech, Gilead, Hikma, Index Pharmaceuticals, Inotrem, Janssen, Lilly, MSD, Mylan, Nestle, Norgine, Oppilan Pharma, OSE Immunotherapeutics, Pandion Therapeutics, Pfizer, Pharmacosmos, Roche, Samsung Bioepis, Sandoz, Sterna, Sublimity Therapeutics, Takeda, Theravance, Tillots, Vifor; grants from AbbVie, Fresenius Kabi, MSD, and Takeda; and stock options from CTMA. Silvio Danese has received consulting fees or honoraria from AbbVie, Allergan, Amgen, AstraZeneca, Biogen, Boehringer Ingelheim, Celgene, Celltrion, Ferring, Gilead, Hospira, Janssen, Johnson \& Johnson, MSD, Mundipharma, and Pfizer. Toshifumi Hibi has received lecture fees from AbbVie, Aspen Japan, Ferring, Gilead, Janssen, JIMRO, Mitsubishi-Tanabe Pharma, Mochida, Pfizer, and Takeda; advisory/consultancy fees from AbbVie, Apo Plus Station, Bristol Myers Squibb, Celltrion, EA Pharma, Eli Lilly, Gilead Sciences, Janssen, Kyorin, Mitsubishi-Tanabe Pharma, Nichi-Iko Pharmaceutical, Pfizer, Takeda, and Zeria; research grants from AbbVie, Activaid, Alfresa Pharma Corporation, Bristol Myers Squibb, Eli Lilly Japan, Ferring, Gilead, Janssen, JMDC, Nippon Kayaku, Mochida, Pfizer Japan, and Takeda; received scholarship contributions from Mitsubishi-Tanabe Pharma, Nippon Kayaku, and Zeria; and belonged to study group sponsorship by AbbVie, EA Pharma,
JIMRO, Kyorin, Mochida, Otsuka, and Zeria. Ken Takeuchi has received consulting fees from Thermo Fisher Diagnostics; research grants from AbbVie and Takeda; and has served as a speaker for AbbVie, Celltrion, EA Pharma, Janssen, Kyorin, Mochida, Takeda, MitsubishiTanabe Pharma, and Zeria. Fernando Magro has served as speaker and received honoraria from AbbVie, Biogen, Falk, Ferring, Hospira, Janssen, Laboratorios Vitoria, MSD, Pfizer, Sandoz, Takeda, UCB, and Vifor. Yoorim An, Dong-Hyeon Kim, and SangWook Yoon are employees of Celltrion Healthcare Co., Ltd. Walter Reinisch has served as a speaker for Abbott Laboratories, AbbVie, Aesca, Aptalis, Astellas, Centocor, Celltrion, Danone Austria, Elan, Falk, Ferring, Immundiagnostik, Mitsubishi-Tanabe Pharma, MSD, Otsuka, PDL, Pharmacosmos, PLS Education, ScheringPlough, Shire, Takeda, Therakos, Vifor, and Yakult; as a consultant for Abbott Laboratories, AbbVie, Aesca, Algernon, Amgen, AM Pharma, AMT, AOP Orphan, Arena Pharmaceuticals, Astellas, AstraZeneca, Avaxia, Bioclinica, Biogen IDEC, Boehringer Ingelheim, Bristol Myers Squibb, Cellerix, Chemocentryx, Celgene, Celltrion, Centocor, Covance, Danone Austria, DSM, Elan, Eli Lilly, Ernst \& Young, Falk, Ferring, Galapagos, Gatehouse Bio, Genentech, Gilead, Grünenthal, ICON, Index Pharma, Inova, Intrinsic Imaging, Janssen, Johnson \& Johnson, Kyowa Hakko Kirin Pharma, Lipid Therapeutics, LivaNova, Mallinckrodt, Medahead, MedImmune, Millennium, MitsubishiTanabe Pharma, MSD, Nash Pharmaceuticals, Nestle, Nippon Kayaku, Novartis, Ocera, OMass, Otsuka, Parexel, PDL, Periconsulting, Pfizer, Pharmacosmos, Philip Morris Institute, Procter \& Gamble, Prometheus, Protagonist, Provention, Quell Therapeutics, Robarts Clinical Trial, Roland Berger, Sandoz, Schering-Plough, Second Genome, Seres Therapeutics, Setpointmedical, Sigmoid, Sublimity, Takeda, Therakos, Theravance, Tigenix, UCB, Vifor, Zealand, Zyngenia, and 4SC; as an advisory board member for Abbott Laboratories, AbbVie, Aesca, Amgen, AM Pharma, Astellas, AstraZeneca, Avaxia, Biogen IDEC, Boehringer Ingelheim, Bristol Myers Squibb, Cellerix, Celgene, Celltrion, Centocor, Chemocentryx, Danone 
Austria, DSM, Elan, Ferring, Galapagos, Genentech, Grünenthal, Inova, Janssen, Johnson \& Johnson, Kyowa Hakko Kirin Pharma, Lipid Therapeutics, MedImmune, Millennium, Mitsubishi-Tanabe Pharma, MSD, Nestle, Novartis, Ocera, Otsuka, PDL, Pfizer, Pharmacosmos, Procter \& Gamble, Prometheus, Sandoz, Schering-Plough, Second Genome, Setpointmedical, Takeda, Therakos, Tigenix, UCB, Zealand, Zyngenia, and 4SC; has received research funding from Abbott Laboratories, AbbVie, Aesca, Centocor, Falk Pharma, Immundiagnostik, Janssen, MSD, Sandoz, and Takeda.

Compliance with Ethics Guidelines. This article is based on previously conducted studies and does not contain any new studies with human participants or animals performed by any of the authors.

Data Availability. Data sharing is not applicable to this article as no datasets were generated or analysed during the current study.

Open Access. This article is licensed under a Creative Commons Attribution-NonCommercial 4.0 International License, which permits any non-commercial use, sharing, adaptation, distribution and reproduction in any medium or format, as long as you give appropriate credit to the original author(s) and the source, provide a link to the Creative Commons licence, and indicate if changes were made. The images or other third party material in this article are included in the article's Creative Commons licence, unless indicated otherwise in a credit line to the material. If material is not included in the article's Creative Commons licence and your intended use is not permitted by statutory regulation or exceeds the permitted use, you will need to obtain permission directly from the copyright holder. To view a copy of this licence, visit http://creativecommons.org/licenses/by$\mathrm{nc} / 4.0 /$.

\section{REFERENCES}

1. Garrido-Cumbrera M, Marzo-Ortega H, Christen L, et al. Assessment of impact of the COVID-19 pandemic from the perspective of patients with rheumatic and musculoskeletal diseases in Europe: results from the REUMAVID study (phase 1). RMD Open. $2021 ; 7(1)$ :e001546.

2. Romão VC, Cordeiro I, Macieira C, et al. Rheumatology practice amidst the COVID-19 pandemic: a pragmatic view. RMD Open. 2020;6(2):e001314.

3. Chebli JMF, Queiroz NSF, Damião A, Chebli LA, Costa MHM, Parra RS. How to manage inflammatory bowel disease during the COVID-19 pandemic: a guide for the practicing clinician. World J Gastroenterol. 2021;27(11):1022-42.

4. Kennedy NA, Jones G-R, Lamb CA, et al. British Society of Gastroenterology guidance for management of inflammatory bowel disease during the COVID-19 pandemic. Gut. 2020;69(6):984-90.

5. Zhou Q, Gao Y, Wang X, et al. Nosocomial infections among patients with COVID-19, SARS and MERS: a rapid review and meta-analysis. Ann Transl Med. 2020;8(10):629.

6. Du Q, Zhang D, Hu W, et al. Nosocomial infection of COVID19: a new challenge for healthcare professionals (review). Int J Mol Med. 2021;47(4):31.

7. Landewé RBM, Machado PM, Kroon F, et al. EULAR provisional recommendations for the management of rheumatic and musculoskeletal diseases in the context of SARS-CoV-2. Ann Rheum Dis. 2020;79(7):851.

8. Magro F, Rahier JF, Abreu C, et al. Inflammatory bowel disease management during the COVID-19 outbreak: the ten do's and don'ts from the ECCOCOVID taskforce. J Crohns Colitis. 2020;14(Suppl 3):S798-806.

9. Akintayo RO, Bahiri R, El Miedany Y, et al. African League Against Rheumatism (AFLAR) preliminary recommendations on the management of rheumatic diseases during the COVID-19 pandemic. Clin Rheumatol. 2021;40(9):3445-54.

10. George MD, Venkatachalam S, Banerjee S, et al. Concerns, healthcare use, and treatment interruptions in patients with common autoimmune rheumatic diseases during the COVID-19 pandemic. J Rheumatol. 2021;48(4):603-7.

11. Parigi TL, D'Amico F, Peyrin-Biroulet L, Danese S. Evolution of infliximab biosimilar in inflammatory bowel disease: from intravenous to subcutaneous CT-P13. Expert Opin Biol Ther. 2021;21(1):37-46. 
12. D'Haens GR, van Deventer S. 25 years of anti-TNF treatment for inflammatory bowel disease: lessons from the past and a look to the future. Gut. 2021;70(7):1396-405.

13. Kim H, Alten R, Cummings $\mathrm{F}$, et al. Innovative approaches to biologic development on the trail of CT-P13: biosimilars, value-added medicines, and biobetters. mAbs. 2021;13(1):1868078.

14. D'Amico F, Solitano V, Aletaha D, et al. Biobetters in patients with immune-mediated inflammatory disorders: an international Delphi consensus. Autoimmun Rev. 2021;20(7):102849.

15. Ahmed M, Bankov G, Casey D, Perry ME. CT-P13 subcutaneous infliximab in gastroenterology and rheumatology. Immunotherapy. 2021;13(12): 1001-9.

16. Verma AM, Patel A, Subramanian S, Smith PJ. From intravenous to subcutaneous infliximab in patients with inflammatory bowel disease: a pandemic-driven initiative. Lancet Gastroenterol Hepatol. 2021;6(2):88-9.

17. Verma A, Patel A. Switching from intravenous to subcutaneous infliximab biosimilar $\left(\right.$ Remsima $\left.^{\circledR}\right)$ in adult patients with inflammatory bowel disease: experience at an NHS trust (sponsored supplement). 2020. https://campus.bsg.org.uk/wp-content/ uploads/2021/01/Celltrion-supplement_Oct-2020PI_digital-version1.pdf. Accessed 14 Oct 2021.

18. Westhovens R, Wiland P, Zawadzki M, et al. Efficacy, pharmacokinetics and safety of subcutaneous versus intravenous CT-P13 in rheumatoid arthritis: a randomized phase I/III trial. Rheumatology (Oxf). 2021;60(5):2277-87.

19. Schreiber S, Ben-Horin S, Leszczyszyn J, et al. Randomized controlled trial: subcutaneous vs intravenous infliximab CT-P13 maintenance in inflammatory bowel disease. Gastroenterology. 2021;160(7):2340-53.

20. Alten R. Benefits of switching from IV to SC infliximab. Presented at EULAR 2021 satellite symposium: management of rheumatic diseases during the COVID-19 pandemic, 3 June 2021.

21. Schreiber S, D'Haens G, Cummings F, et al. Switching from intravenous to subcutaneous infliximab in patients with active inflammatory bowel disease: post-hoc analysis of pre/post switch outcomes from a multicentre, randomised controlled pivotal trial [P0472, presented at UEG Week 2021]. https://programme.ueg.eu/week2021/\#/ details/presentations/1351. Accessed 14 Oct 2021.

22. Argüelles-Arias F, Fernández Álvarez P, Castro Laria $\mathrm{L}$, et al. Switch to infliximab subcutaneous during
SARS-CoV-2 pandemic: preliminary results. Rev Esp Enferm Dig. 2021. 2022;114(2):118-9.

23. Al Sulais E, AlAmeel T. Biosimilars to antitumor necrosis factor agents in inflammatory bowel disease. Biologics. 2020;14:1-11.

24. Melsheimer R, Geldhof A, Apaolaza I, Schaible T. Remicade $^{\circledR}$ (infliximab): 20 years of contributions to science and medicine. Biologics. 2019;13:139-78.

25. Smolen JS, Emery P. Infliximab: 12 years of experience. Arthritis Res Ther. 2011;13:S2.

26. Vezér B, Buzás Z, Sebeszta M, Zrubka Z. Authorized manufacturing changes for therapeutic monoclonal antibodies (mAbs) in European Public Assessment Report (EPAR) documents. Curr Med Res Opin. 2016;32(5):829-34.

27. Vanderlaan M, Maniatis A, Olney R, et al. Changes in manufacturing processes of biologic therapies can alter the immunogenicity profile of the product. Clin Pharmacol Ther. 2020;107(4):988-93.

28. Raffals LE, Nguyen GC, Rubin DT. Switching between biologics and biosimilars in inflammatory bowel disease. Clin Gastroenterol Hepatol. 2019;17(5):818-23.

29. Müller-Ladner U, Hong S, Oh C, Taylor P. Scientific rationale behind the development and approval of biosimilar infliximab (CT-P13) in Europe. Expert Rev Clin Immunol. 2015;11(Suppl 1):S5-14.

30. Generics and Biosimilar Initiative. Biosimilars approved in Japan. 2014. https://gabionline.net/ biosimilars/general/Biosimilars-approved-in-Japan. Accessed 14 Oct 2021.

31. Generics and Biosimilar Initiative. Biosimilars approved in South Korea. 2014. https://www. gabionline.net/biosimilars/general/Biosimilarsapproved-in-South-Korea. Accessed 14 Oct 2021.

32. Park W, Hrycaj P, Jeka S, et al. A randomised, double-blind, multicentre, parallel-group, prospective study comparing the pharmacokinetics, safety, and efficacy of CT-P13 and innovator infliximab in patients with ankylosing spondylitis: the PLANETAS study. Ann Rheum Dis. 2013;72(10):1605-12.

33. Yoo DH, Hrycaj P, Miranda P, et al. A randomised, double-blind, parallel-group study to demonstrate equivalence in efficacy and safety of CT-P13 compared with innovator infliximab when coadministered with methotrexate in patients with active rheumatoid arthritis: the PLANETRA study. Ann Rheum Dis. 2013;72(10):1613-20.

34. Ye BD, Pesegova M, Alexeeva O, et al. Efficacy and safety of biosimilar CT-P13 compared with 
originator infliximab in patients with active Crohn's disease: an international, randomised, double-blind, phase 3 non-inferiority study. Lancet. 2019;393(10182):1699-707.

35. European Medicines Agency. Remsima: assessment report on extension(s) of marketing authorisation. 2019. https://www.ema.europa.eu/en/documents/ variation-report/remsima-h-c-2576-X-0062-eparassessment-report-variation_en.pdf. Accessed 14 Oct 2021.

36. European Medicines Agency. Remsima summary of product characteristics. 2021. https://www.ema. europa.eu/en/documents/product-information/ remsima-epar-product-information_en.pdf. Accessed 14 Oct 2021.

37. European Medicines Agency. Remsima: summary of opinion (post-authorisation). 2020. https://www. ema.europa.eu/en/documents/smop/chmp-postauthorisation-summary-positive-opinion-remsimaii/82_en.pdf. Accessed 14 Oct 2021.

38. Health Canada. Remsima SC: product monograph including patient medication information. 2021. https://pdf.hres.ca/dpd_pm/00059823.PDF. Accessed 14 Oct 2021.

39. Joachim R. Subcutaneous infliximab in the U.S.: defining CT-P13 SC's competitive edge. 2021. https://www.biosimilardevelopment.com/doc/ subcutaneous-infliximab-in-the-u-s-defining-ct-psc-s-competitive-edge-0001. Accessed 14 Oct 2021.

40. European Medicines Agency. Remsima: procedural steps taken and scientific information after the authorisation. 2021. https://www.ema.europa.eu/ en/documents/procedural-steps-after/remsimaepar-procedural-steps-taken-scientific-informationafter-authorisation_en.pdf. Accessed 14 Oct 2021.

41. Atiqi S, Hooijberg F, Loeff FC, Rispens T, Wolbink GJ. Immunogenicity of TNF-inhibitors. Front Immunol. 2020;11:312.

42. Bittner B, Richter W, Schmidt J. Subcutaneous administration of biotherapeutics: an overview of current challenges and opportunities. BioDrugs. 2018;32(5):425-40.

43. Yoo D, Westhovens R, Ben-Horin S, et al. Development of a subcutaneous formulation of CT-P13 (infliximab): maintenance subcutaneous administration may elicit lower immunogenicity compared to intravenous treatment. Arthritis Rheumatol. 2018;70(Suppl 9):2791-2.

44. Bar-Yoseph H, Pressman S, Blatt A, et al. Infliximab-tumor necrosis factor complexes elicit formation of anti-drug antibodies. Gastroenterology. 2019;157(5):1338-51.e8.
45. Combe B, Allanore Y, Alten R, et al. Comparative efficacy of subcutaneous (CT-P13) and intravenous infliximab in adult patients with rheumatoid arthritis: a network meta-regression of individual patient data from two randomised trials. Arthritis Res Ther. 2021;23(1):119.

46. Caporali R, Allanore Y, Alten R, et al. Efficacy and safety of subcutaneous infliximab versus adalimumab, etanercept and intravenous infliximab in patients with rheumatoid arthritis: a systematic literature review and meta-analysis. Expert Rev Clin Immunol. 2021;17(1):85-99.

47. Shah RB, Patel M, Maahs DM, Shah VN. Insulin delivery methods: past, present and future. Int J Pharm Investig. 2016;6(1):1-9.

48. Striesow F, Brandt A. Preference, satisfaction and usability of subcutaneously administered methotrexate for rheumatoid arthritis or psoriatic arthritis: results of a postmarketing surveillance study with a high-concentration formulation. Ther Adv Musculoskelet Dis. 2012;4(1):3-9.

49. Allen PB, Lindsay H, Tham TC. How do patients with inflammatory bowel disease want their biological therapy administered? BMC Gastroenterol. 2010;10:1.

50. Dehoratius RJ, Brent LH, Curtis JR, Ellis LA, Tang KL. Satisfaction with subcutaneous golimumab and its auto-injector among rheumatoid arthritis patients with inadequate response to adalimumab or etanercept. Patient. 2018;11(3):361-9.

51. Reinisch W, Gecse K, Halfvarson J, et al. Clinical practice of adalimumab and infliximab biosimilar treatment in adult patients with Crohn's disease. Inflamm Bowel Dis. 2021;27(1):106-22.

52. PharmaTimes. Celltrion bags EU approval for Remsima SC off back of new data. 2019. http://www. pharmatimes.com/news/celltrion_bags_eu_ approval_for_remsima_sc_off_back_of_new_data 1318170. Accessed 14 Oct 2021.

53. Huynh TK, Ostergaard A, Egsmose C, Madsen OR. Preferences of patients and health professionals for route and frequency of administration of biologic agents in the treatment of rheumatoid arthritis. Patient Prefer Adherence. 2014;8:93-9.

54. Mizoshita T, Katano T, Tanida S, et al. Prospective comparison of preference and efficacy of adalimumab and infliximab for treating ulcerative colitis naive to antitumor necrosis factor therapy. Medicine (Baltimore). 2017;96(32):e7800.

55. Vavricka SR, Bentele N, Scharl M, et al. Systematic assessment of factors influencing preferences of Crohn's disease patients in selecting an anti-tumor 
necrosis factor agent (CHOOSE TNF TRIAL). Inflamm Bowel Dis. 2012;18(8):1523-30.

56. Aluzaite K, Greveson K, Ben-Horin S, Leong R, Haj $\mathrm{O}$, Schultz M. Barriers to international travel in inflammatory bowel disease patients. J Travel Med. 2021;28(1):taaa197.

57. IBD Passport. Receiving biologic drugs overseas. https://www.ibdpassport.com/health-care-abroad. Accessed 14 Oct 2021.

58. Arthritis Australia. Things to consider when taking a biologic. https://arthritisaustralia.com.au/thingsto-consider-when-taking-a-biologic/. Accessed 14 Oct 2021.

59. Mikuls TR, Johnson SR, Fraenkel L, et al. American College of Rheumatology guidance for the management of rheumatic disease in adult patients during the COVID-19 pandemic: version 3. Arthritis Rheumatol. 2021;73(2):e1-12.

60. National Institute for Health and Care Excellence. COVID-19 rapid guideline: rheumatological autoimmune, inflammatory and metabolic bone disorders. 2020. https://www.nice.org.uk/guidance/ ng167/resources/covid19-rapid-guidelinerheumatological-autoimmune-inflammatory-andmetabolic-bone-disorders-pdf-66141905788357. Accessed 14 Oct 2021.

61. National Institute for Health and Care Excellence. COVID-19 rapid guideline: gastrointestinal and liver conditions treated with drugs affecting the immune response. 2020. https://www.nice.org.uk/ guidance/ng172/resources/covid19-rapid-guidelinegastrointestinal-and-liver-conditions-treated-withdrugs-affecting-the-immune-response-pdf66141957856453 . Accessed 14 Oct 2021.

62. Richez C, Flipo R-M, Berenbaum F, et al. Managing patients with rheumatic diseases during the COVID-19 pandemic: the French Society of Rheumatology answers to most frequently asked questions up to May 2020. Jt Bone Spine. 2020;87(5):431-7.

63. Rubin DT, Abreu MT, Rai V, et al. Management of patients with Crohn's disease and ulcerative colitis during the coronavirus disease-2019 pandemic: results of an international meeting. Gastroenterology. 2020;159(1):6-13.e6.

64. Rubin DT, Feuerstein JD, Wang AY, Cohen RD. AGA clinical practice update on management of inflammatory bowel disease during the COVID-19 pandemic: expert commentary. Gastroenterology. 2020;159(1):350-7.

65. Zhu L-R, Mao R, Fiorino G, Schneider T. 2nd Interview COVID-19 ECCO Taskforce. 2020. https://
ecco-ibd.eu/images/6_Publication/6_8_Surveys/ 2nd_Interview_COVID-19_ECCO_Taskforce_ published.pdf. Accessed 14 Oct 2021.

66. Van Assche G, Vermeire S, Ballet V, et al. Switch to adalimumab in patients with Crohn's disease controlled by maintenance infliximab: prospective randomised SWITCH trial. Gut. 2012;61(2):229-34.

67. Combier A, Wanono S, Poiroux L, et al. Switching tocilizumab or abatacept from the intravenous to the subcutaneous route during the COVID-19 pandemic: French experience [in French]. Rev Rhum Ed Fr. 2021;88(6):471-2.

68. Gupta R, Shipa M, Yeoh SA, Buck P, Ehrenstein MR. An unfavourable outcome following switching intravenous abatacept and tocilizumab to subcutaneous forms during the COVID-19 pandemic. Rheumatology (Oxf). 2021;60(2):977-9.

69. Solitano V, Vuitton L, Peyrin-Biroulet L, Danese S. The evolution of biologics administration from intravenous to subcutaneous: treatments for inflammatory bowel disease go home. Gastroenterology. 2021;160(7):2244-7.

70. Smith PJ, Storey D, Gregg B, et al. Efficacy and safety of elective switching of inflammatory bowel disease patients from intravenous to subcutaneous infliximab (IFX): a multi-centre cohort study (P496). J Crohns Colitis. 2021;15(Suppl 1):S480-1.

71. Schreiber S, Ben-Horin S, Reinisch W, Vavricka S. Clinical benefits of anti-TNF therapy for the management of IBD: biologic and biosimilar drugs. 2021. https://www.medscape.org/viewarticle/ 951384. Accessed 14 Oct 2021.

72. Larsson I, Bergman S, Fridlund B, Arvidsson B. Patients' independence of a nurse for the administration of subcutaneous anti-TNF therapy: a phenomenographic study. Int J Qual Stud Health WellBeing. 2010. https://doi.org/10.3402/qhw.v5i2. 5146.

73. Malm K, Bergman S, Andersson ML, Bremander A, Larsson I. Quality of life in patients with established rheumatoid arthritis: a phenomenographic study. SAGE Open Med. 2017;5:2050312117713647.

74. Heald A, Bramham-Jones S, Davies M. Comparing cost of intravenous infusion and subcutaneous biologics in COVID-19 pandemic care pathways for rheumatoid arthritis and inflammatory bowel disease: a brief UK stakeholder survey. Int J Clin Pract. 2021;75(9):e14341.

75. Perry M, Jang M. Budget impact analysis of introducing subcutaneous infliximab CT-P13 SC from the UK payer perspective (AB1185). Ann Rheum Dis. 2020;79:1879. 
76. Ungaro RC, Brenner EJ, Gearry RB, et al. Effect of IBD medications on COVID-19 outcomes: results from an international registry. Gut. 2021;70(4):725.

77. Akiyama S, Hamdeh S, Micic D, Sakuraba A. Prevalence and clinical outcomes of COVID-19 in patients with autoimmune diseases: a systematic review and meta-analysis. Ann Rheum Dis. 2021;80(3):384.

78. Brenner EJ, Ungaro RC, Gearry RB, et al. Corticosteroids, but not TNF antagonists, are associated with adverse COVID-19 outcomes in patients with inflammatory bowel diseases: results from an international registry. Gastroenterology. 2020;159(2):481-91.e3.

79. Wang Q, Liu J, Shao R, Han X, Su C, Lu W. Risk and clinical outcomes of COVID-19 in patients with rheumatic diseases compared with the general population: a systematic review and meta-analysis. Rheumatol Int. 2021;41(5):851-61.

80. Brito CA, Paiva JG, Pimentel FN, Guimarães RS, Moreira MR. COVID-19 in patients with rheumatological diseases treated with anti-TNF. Ann Rheum Dis. 2021;80(5):e62.

81. Scribano ML. Why do immunosuppressed patients with inflammatory bowel disease not seem to be at a higher risk of COVID-19? Dig Dis Sci. 2021;66(9): 2855-64.

82. Gianfrancesco M, Hyrich KL, Al-Adely $\mathrm{S}$, et al. Characteristics associated with hospitalisation for COVID-19 in people with rheumatic disease: data from the COVID-19 Global Rheumatology Alliance physician-reported registry. Ann Rheum Dis. 2020;79(7):859-66.

83. Stallmach A, Kortgen A, Gonnert F, Coldewey SM, Reuken $\mathrm{P}$, Bauer $\mathrm{M}$. Infliximab against severe COVID-19-induced cytokine storm syndrome with organ failure-a cautionary case series. Crit Care. 2020;24(1):444.

84. Kennedy NA, Lin S, Goodhand JR, et al. Infliximab is associated with attenuated immunogenicity to BNT162b2 and ChAdOx1 nCoV-19 SARS-CoV-2 vaccines in patients with IBD. Gut. 2021;70(10): 1884-93.

85. D'Haens G, Reinisch W, Schreiber S, et al. Comparison of combination subcutaneous infliximab and an immunomodulator versus subcutaneous infliximab monotherapy: post-hoc analysis of a randomised clinical trial [P0467, presented at UEG Week 2021]. https://programme.ueg.eu/week2021/ \#/details/presentations/1284. Accessed 14 Oct 2021.

86. Colombel J-F, Adedokun OJ, Gasink C, et al. Combination therapy with infliximab and azathioprine improves infliximab pharmacokinetic features and efficacy: a post hoc analysis. Clin Gastroenterol Hepatol. 2019;17(8):1525-32.e1.

87. Burgueño JF, Reich A, Hazime $\mathrm{H}$, et al. Expression of SARS-CoV-2 entry molecules ACE2 and TMPRSS2 in the gut of patients with IBD. Inflamm Bowel Dis. 2020;26(6):797-808.

88. Ardizzone S, Ferretti F, Monico MC, et al. Lower incidence of COVID-19 in patients with inflammatory bowel disease treated with non-gut selective biologic therapy. J Gastroenterol Hepatol. 2021;36(11):3050-5.

89. Robinson PC, Richards D, Tanner HL, Feldmann M. Accumulating evidence suggests anti-TNF therapy needs to be given trial priority in COVID-19 treatment. Lancet Rheumatol. 2020;2(11):e653-5.

90. World Health Organization. WHO's Solidarity clinical trial enters a new phase with three new candidate drugs. 2021. https://www.who.int/news/ item/11-08-2021-who-s-solidarity-clinical-trialenters-a-new-phase-with-three-new-candidatedrugs. Accessed 14 Oct 2021.

91. Li Y, Zhao W, Liu J, Chen Z, Lv Q, Zhang Z. Immunotherapy summary for cytokine storm in COVID-19. Front Pharmacol. 2021;12:731847.

92. Mornese Pinna S, Lupia T, Scabini S, et al. Monoclonal antibodies for the treatment of COVID-19 patients: an umbrella to overcome the storm? Int Immunopharmacol. 2021;101(Pt A):108200.

93. Gatti M, Turrini E, Raschi E, Sestili P, Fimognari C. Janus kinase inhibitors and coronavirus disease (COVID)-19: rationale, clinical evidence and safety issues. Pharmaceuticals (Basel). 2021;14(8):738.

94. Florescu DF, Kalil AC. Janus kinase inhibitors for the treatment of hospitalized patients with COVID-19. Curr Opin Crit Care. 2021;27(5):493-6.

95. Izadi Z, Brenner EJ, Mahil SK, et al. Association between tumor necrosis factor inhibitors and the risk of hospitalization or death among patients with immune-mediated inflammatory disease and COVID-19. JAMA Netw Open. 2021;4(10): e2129639.

96. Businesswire. Celltrion's monoclonal antibody treatment for COVID-19, regdanvimab (CT-P59) becomes the first authorized COVID-19 treatment approved from the Korean Ministry of Food and Drug Safety (MFDS). 2021. https://www. businesswire.com/news/home/20210918005026/ en/\%C2\%A0Celltrion\%E2\%80\%99s-monoclonalantibody-treatment-for-COVID-19-regdanvimabCT-P59-becomes-the-first-authorized-COVID-19- 
treatment-approved-from-the-Korean-Ministry-ofFood-and-Drug-Safety-MFDS. Accessed 14 Oct 2021.

97. Chanchlani N, Lin S, Chee D, et al. Adalimumab and infliximab impair SARS-CoV-2 antibody responses: results from a therapeutic drug monitoring study in 11422 biologic-treated patients. J Crohns Colitis. 2021. https://doi.org/10.1093/ ecco-jcc/jjab153.

98. Mahil SK, Bechman K, Raharja A, et al. The effect of methotrexate and targeted immunosuppression on humoral and cellular immune responses to the COVID-19 vaccine BNT162b2: a cohort study. Lancet Rheumatol. 2021;3(9):e627-37.

99. Lee SM, Lee D. Opportunities and challenges for contactless healthcare services in the post-COVID19 era. Technol Forecast Soc Change. 2021;167: 120712.

100. Nune A, Iyengar K, Ahmed A, Sapkota H. Challenges in delivering rheumatology care during COVID-19 pandemic. Clin Rheumatol. 2020;39(9): 2817-21.

101. Lewin S, Lees C, Regueiro M, Hart A, Mahadevan U. International Organization for the Study of Inflammatory Bowel Disease: global strategies for telemedicine and inflammatory bowel diseases. J Crohns Colitis. 2020;14(Suppl 3):S780-4.

102. Gonzalez HA, Myers S, Whitehead E, et al. React, reset and restore: adaptation of a large inflammatory bowel disease service during COVID-19 pandemic. Clin Med (Lond). 2020;20(5):e183-8.

103. Matsumoto RA, Barton JL. Telerheumatology: before, during, and after a global pandemic. Curr Opin Rheumatol. 2021;33(3):262-9.

104. Aguas M, Del Hoyo J, Faubel R, Nos P. Use of telemedicine in inflammatory bowel disease: a real monitoring option? Expert Rev Gastroenterol Hepatol. 2016;10(8):879-81.

105. Kernder A, Morf H, Klemm P, et al. Digital rheumatology in the era of COVID-19: results of a national patient and physician survey. RMD Open. 2021;7(1):e001548.

106. Piga M, Cangemi I, Mathieu A, Cauli A. Telemedicine for patients with rheumatic diseases: systematic review and proposal for research agenda. Semin Arthritis Rheum. 2017;47(1):121-8.

107. Kong SS, Otalora Rojas LA, Ashour A, Robinson M, Hosterman T, Bhanusali N. Ability and willingness to utilize telemedicine among rheumatology patients-a cross-sectional survey. Clin Rheumatol. 2021;40(12):5087-93.
108. Chevallard M, Belloli L, Ughi N, et al. Use of telemedicine during the COVID-19 pandemic in patients with inflammatory arthritis: a retrospective study on feasibility and impact on patient-reported outcomes in a real-life setting. Rheumatol Int. 2021;41(7):1253-61.

109. George MD, Baker JF, Banerjee S, et al. Social distancing, health care disruptions, telemedicine use, and treatment interruption during the COVID-19 pandemic in patients with or without autoimmune rheumatic disease. ACR Open Rheumatol. 2021;3(6):381-9.

110. Cross RK, Langenberg P, Regueiro M, et al. A randomized controlled trial of TELEmedicine for Patients with Inflammatory Bowel Disease (TELEIBD). Am J Gastroenterol. 2019;114(3):472-82.

111. de Jong MJ, van der Meulen-de Jong AE, RombergCamps MJ, et al. Telemedicine for management of inflammatory bowel disease (myIBDcoach): a pragmatic, multicentre, randomised controlled trial. Lancet. 2017;390(10098):959-68.

112. Kennedy NA, Hansen R, Younge L, et al. Organisational changes and challenges for inflammatory bowel disease services in the UK during the COVID19 pandemic. Frontline Gastroenterol. 2020;11(5): 343-50.

113. Kataria S, Ravindran V. Digital health: a new dimension in rheumatology patient care. Rheumatol Int. 2018;38(11):1949-57.

114. Yin AL, Hachuel D, Pollak JP, Scherl EJ, Estrin D. Digital health apps in the clinical care of inflammatory bowel disease: scoping review. J Med Internet Res. 2019;21(8):e14630.

115. Colombel JF, Panaccione R, Bossuyt P, et al. Effect of tight control management on Crohn's disease (CALM): a multicentre, randomised, controlled phase 3 trial. Lancet. 2017;390(10114):2779-89.

116. Bastida C, Ruíz V, Pascal M, Yagüe J, Sanmartí R, Soy D. Is there potential for therapeutic drug monitoring of biologic agents in rheumatoid arthritis? Br J Clin Pharmacol. 2017;83(5):962-75.

117. Vande Casteele N, Feagan BG, Wolf DC, et al. Therapeutic drug monitoring of tumor necrosis factor antagonists in Crohn disease: a theoretical construct to apply pharmacokinetics and guidelines to clinical practice. Inflamm Bowel Dis. 2021;27(8): 1346-55.

118. Ungar B, Engel T, Yablecovitch D, et al. Prospective observational evaluation of time-dependency of adalimumab immunogenicity and drug concentrations: the POETIC study. Am J Gastroenterol. 2018;113(6):890-8. 
119. Ward MG, Thwaites PA, Beswick L, et al. Intra-patient variability in adalimumab drug levels within and between cycles in Crohn's disease. Aliment Pharmacol Ther. 2017;45(8):1135-45.

120. Ganesananthan S, Durai D. Clinical value and cost saving of therapeutic drug monitoring of infliximab in adult patients with inflammatory bowel disease. Clin Med (Lond). 2020;20(Suppl 2):s23-4.

121. Guidi L, Pugliese D, Panici Tonucci T, et al. Therapeutic drug monitoring is more cost-effective than a clinically based approach in the management of loss of response to infliximab in inflammatory bowel disease: an observational multicentre study. J Crohns Colitis. 2018;12(9):1079-88.

122. Cea-Calvo L, Carmona L, Calvo-Alén J. The challenge of assessing adherence to subcutaneous biological drugs in immune-mediated inflammatory diseases. Letter to the editor regarding Michetti $\mathrm{P}$, Weinman J, Mrowietz U, et al. Adv Ther (2017);34: 91-108. doi:10.1007/s12325-016-0441-3. Adv Ther. 2017;34(9):2173-6.
123. Fayet F, Fan A, Rodere M, Savel C, Pereira B, Soubrier $\mathrm{M}$. Adherence to subcutaneous anti-TNF treatment in chronic inflammatory rheumatism and therapeutic patient education. Patient Prefer Adherence. 2020;14:363-9.

124. Bhoi P, Bessette L, Bell MJ, Tkaczyk C, Nantel F, Maslova K. Adherence and dosing interval of subcutaneous antitumour necrosis factor biologics among patients with inflammatory arthritis: analysis from a Canadian administrative database. BMJ Open. 2017;7(9):e015872.

125. Bonek K, Roszkowski L, Massalska M, Maslinski W, Ciechomska M. Biologic drugs for rheumatoid arthritis in the context of biosimilars, genetics, epigenetics and COVID-19 treatment. Cells. 2021;10(2):323.

126. Pombo-Suarez M, Maneiro Fernandez JR, GomezReino JJ. Adherence to treatment in patients with rheumatoid arthritis from Spain. Patient Prefer Adherence. 2021;15:111-7. 